\title{
HIGH POWER TEST OF THE FIRST C-BAND (5712 MHz) 50 MW PPM KLYSTRON
}

\author{
H. Matsumoto ${ }^{\dagger}$, T. Shintake, KEK, Tsukuba, Ibaraki, 305-0801 Japan \\ Y. Ohkubo, H. Taoka, Toshiba Co., Ohtawara, Tochigi, 324-8550 Japan \\ K. Ohhashi and K. Kakuno, Shin-Etsu Chemical Co., Ltd, Takefu, Fukui, 915-8515 Japan
}

\section{Abstract}

Hot isostatic pressing (HIP) technique has been firstly applied to fabricate a magnetic circuit in a PPM klystron. Simply stacking disks of the magnetic stainless-steel (Mag-SUS) and oxygen-free-copper (OFC) alternatively, and processing in a HIP vessel filled with pressurised Argas at $1200-\mathrm{kgf} / \mathrm{cm}^{2}$ and temperature of $800-{ }^{\circ} \mathrm{C}$ for 2 hours, they were bonded in one block with diffusion bonding. No brazing-alloys were used in this process. After machining the rf-cavities and beam drift-tube on the bonded PPM stack, they were assembled together in one body by means of conventional brazing method.

The C-band PPM klystron based on this technique was fabricated in the course of the Linear Collider R\&D. Output power of $37 \mathrm{MW}$ was generated with $2.5-\mu \mathrm{sec}$ pulse width and 50 pps repetition rate.

\section{INTRODUCTION}

The Phase-I (500-GeV C.M. energy) JLC project requires $3500 \mathrm{C}$-band klystrons of 50-MW output power level [2], [3]. We started R\&D on C-band RF system development in 1996, since then we have developed three 50-MW class klystrons in three years (1996-1998). All of them successfully generated rf output power of 50-MW or higher. After long-run life-test ( $>5000$ hours each), we confirmed the developed klystrons were acceptable for the JLC-I project [3].

All of them used the electrical solenoid magnet for beam focusing, which dissipates electrical power of $5 \mathrm{~kW}$ This power is quite small as compared with total system power consumption. However, by replacing the focusing solenoid with a permanent magnet, we can eliminate the solenoid magnet itself, its DC power supply, the water cooling system and its interlock, thus the system becomes quite simple.

The PPM-focusing scheme was firstly applied to a high-power klystron by D. Sprehn [4] in 1996 at SLAC in the course of R\&D for the X-band NLC. The PPM Xband klystron demonstrated excellent performance at an output power level over 50-MW and a beam-to-rf powerefficiency as high as $60 \%$. In spite of this promising result, industrialization of this type of the tube seems to be not easy, because (1) we have to integrate the relatively complicated magnetic circuits into the vacuum tube; at least 20 pole-pieces are required in one tube and (2) the polepieces are usually made of iron which is troublesome in the brazing process due to difference in thermal expansion coefficient between iron and copper, and also the †hiroshi.matsumoto@kek.jp

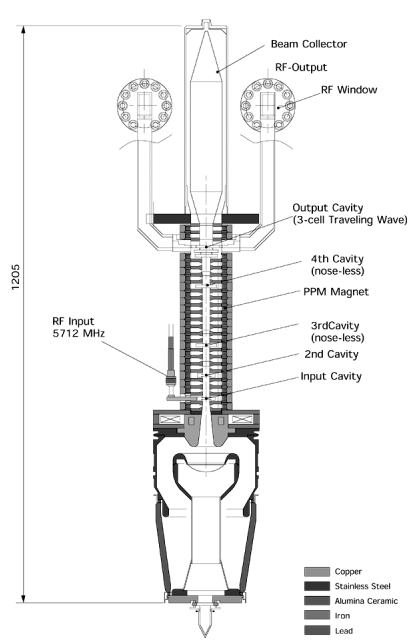

Fig. 1: Cut away view of the first C-band (5712-MHz) $50 \mathrm{MW}-$ class PPM type klystrons (TOSHIBA-E3747).

we decided to upgrade the existing design of our C-band klystron (TOSHIBA E3746 No.3-tube, solenoid focus). We kept the same design in the electron gun, the output cavity (travellingwave structure) and the beam collector. Only the drifttube part was renewed in order to implement the PPM focusing scheme.

Table 1: Main target parameters

\begin{tabular}{|l|rl|}
\hline Frequency & 5712 & $\mathrm{MHz}$ \\
\hline Output rf power & 50 & $\mathrm{MW}$ \\
\hline RF pulse width & 2.5 & $\mu \mathrm{sec}$ \\
\hline Beam voltage & 350 & $\mathrm{KV}$ \\
\hline Beam current & 317 & $\mathrm{~A}$ \\
\hline RF power efficiency & 48 & $\%$ \\
\hline Perveance $\left(10^{-6}\right)$ & 1.53 & $\mathrm{~A} / \mathrm{V}^{1.5}$ \\
\hline Drift tube radius (up/down stream) & $7.5 / 9.0$ & $\mathrm{~mm}$ \\
\hline Beam radius (up stream) & 5.0 & $\mathrm{~mm}$ \\
\hline Brillouin field (relativistic) & 1.9 & $\mathrm{kG}$ \\
\hline Peak field (on axis, upstream) & 2.0 & $\mathrm{kG}$ \\
\hline Plasma wavelength $(\lambda)$ & 82.3 & $\mathrm{~mm}$ \\
\hline PPM pitch $(\lambda)$ & 30 & $\lambda . \mathrm{PPM}$ \\
\hline Cutoff voltage $(\alpha=66)$ & 23 & $\mathrm{kV}$ \\
\hline Permanent Magnet 1$)$ & 1.2 & $\mathrm{Tesla}$ \\
& 11 & $\mathrm{k}-\mathrm{Oe}$ \\
Residual Induction: $\mathrm{Br}$ & Neodymium & $\mathrm{N} 40 \mathrm{~A}$ \\
Coercive force: $\mathrm{Hc}$ & Mag-SUS & \\
\hline Pole-piece & Fe+14\%Cr+C $(20-p p m)$ \\
\hline
\end{tabular}

\subsection{Electrical Design}

We use the FCI-code [5] to find the optimum magneticfield profile. FCI simulates the beam dynamics in the drift-tube based the PIC-method, which takes into account the space-charge field, interaction with RF-cavities, and 
the external focusing PPM-field. The target electrical parameters are listed in Table 1.

\subsection{Design of PPM Focusing System}

We chose "Mag-SUS" for the pole-piece material instead of pure iron. The principal chemical composition is $\mathrm{Fe}+14 \% \mathrm{Cr}$, which eliminates the rust problem. The carbon content is controlled below 20-ppm; this is important in preserving the ferrite-phase after the heat-cycles during the HIP and conventional brazing processes.

We chose the neodymium magnet $(\mathrm{Nd} 2 \mathrm{Fe} 14 \mathrm{~B})$ as the
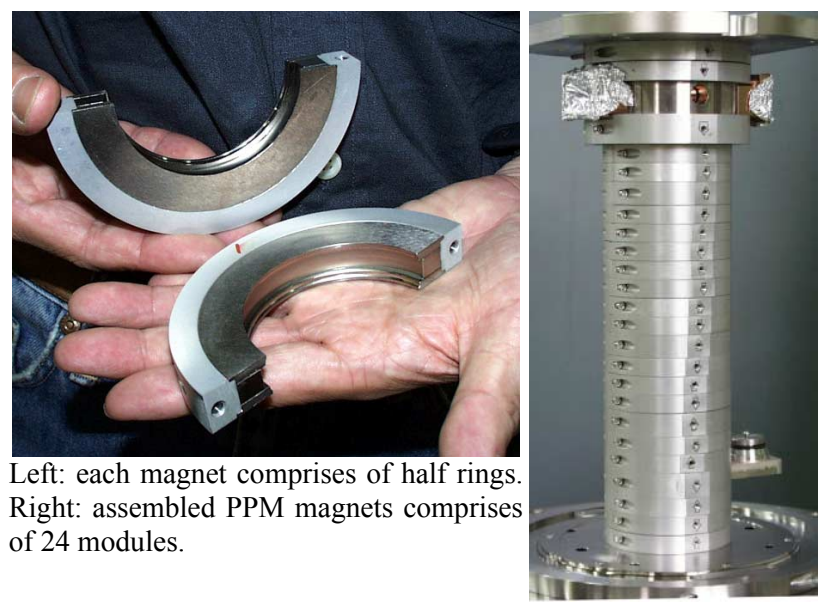

Figure 2: PPM magnet mounted on the main body of the klystron.

permanent magnet, because of its excellent magnetic performance. Half-ring shape neodymium magnet is sandwiched between two iron pole-pieces and moulded to aluminium guard ring as shown in Fig. 2. After assembling those parts in one module using "Epoxy-Bond", the magnet was magnetized.

Fig. 3 shows the simulated magnetic-field patterns. Since the Mag-SUS disks are faced in parallel with relatively wide area, a large amount of the magnetic field flux is bypassed between them. In this design, while the field utilization efficiency is low, it provides a fairly stable magnetic field inside the drift-tube. Additionally, the thick body serves as a radiation shield for the neodymium magnet, and provides space for the cooling water channel and enough mechanical rigidity to support the electron gun narts

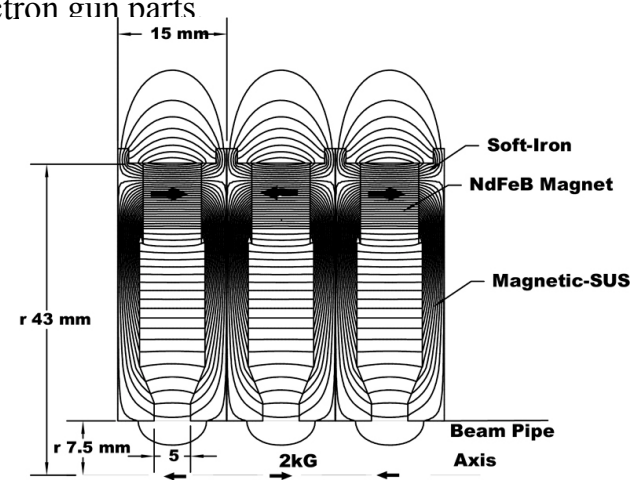

Figure 3: Cutaway view of the PPM structure and the magneticfield pattern. electron gun parts.

Before assembling the electron gun and beam collector, all of the permanent magnet elements were mounted on the klystron body as seen in Fig 2, and the magnetic field was measured. Fig. 4 shows the measured field profile along the beam axis. The large positive swing around $\mathrm{z}=550 \mathrm{~mm}$ corresponds to the output circuit of the threecell travelling wave structure. The measured data is within tolerance of the expected profile.

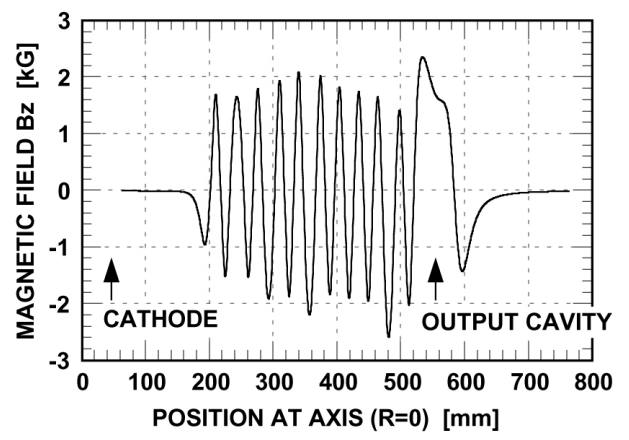

Figure 4: Measured field profile along the axis at the center.

\section{FABRICATION OF PPM CIRCUIT}

\subsection{Diffusion Bonding}

In order to carry the magnetic flux from the permanent magnet to the drift tube, the periodic magnetic-circuit is required. We employ the integrated magnetic circuit design, i.e., stack of copper and Mag-SUS disk provides both the magnetic circuit and the vacuum envelop of the klystron, at the same time. To make the stack, we employed the HIP process [6] as follows:

(1) Prepare disks made of "Mag-SUS" and OFC copper with purity better than $99.99 \%$.

(2) Stack them alternatively, without brazing alloy.

(3) Put them in a vacuum capsule made of OFC copper as shown in Fig. 5, and seal up both end-caps by the electron-beam welder (EBW) in vacuum.

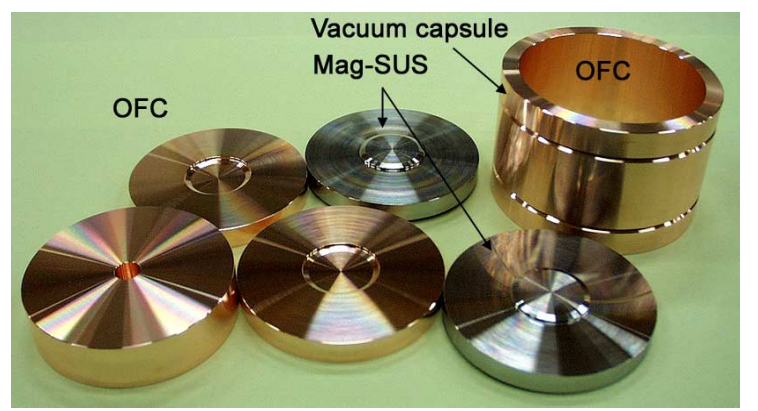

Figure 5: Machined parts pf PPM stack before assembly. They are stacked together and loaded into the vacuum capsule.

(4) Process in HIP at high pressure of $1200-\mathrm{kgf} / \mathrm{cm}^{2}$ and high temperature of $800-{ }^{\circ} \mathrm{C}$ for 2 hours in Ar-gas. Fig. 6 shows a schematic layout of HIP process. (5) Remove the capsule part from the bonded block on the turning lathe. Drill the beam hole on the axis, and four water channels around. Machine the rf-cavity and the waveguide port. 


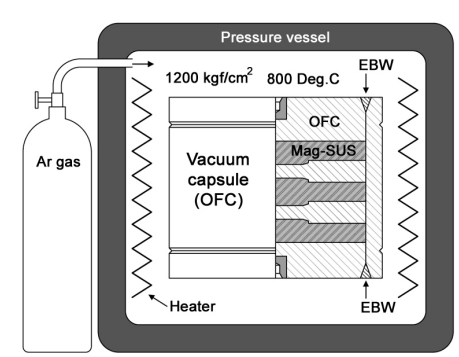

Figure 6: HIP diffusion bonding process. metal-to-metal bonding, even if the pair of materials has different physical properties [6]. In the present case, the thermal expansion coefficients are quite different in two (Mag-SUS is $1.2 \times 10^{-5}$, and the copper is $1.7 \times 10^{-5}$ ). Even through, the bonding process was performed very well with HIP. We believe that the external force applied

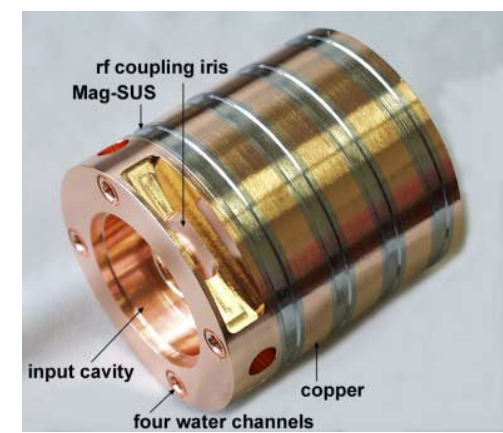

Figure 7: The diffusion bonded PPM stack. The input rf-cavity, a coupling iris, the beam hole and four water cooling channels were machined after the HIP bonding. with the Ar-gas holds the materials stably during the HIP process, and suppresses deformation or slip associated with the difference in thermal expansions (possibly plastic-flow in copper also helps this process). A schematic view of the HIP process is shown in Fig. 6.

\section{TEST RESULTS}

Fig. 8 shows waveforms of the rf output power, the beam voltage and input drive power. The first C-band PPM klystron generated 37-MW output power at the beam voltage of $350-\mathrm{kV}$, pulse width of $2.5-\mu \mathrm{sec}$ and repetition rate of $50 \mathrm{pps}$.

The output power was measured by the absolute calorimetric method, which comprises of the rf-water-load, an electrical-hater module and four precise temperature sensors. The output power was determined from the temperature rise at the water loads. This power measurement system is calibrated by temperature rise at the electrical-

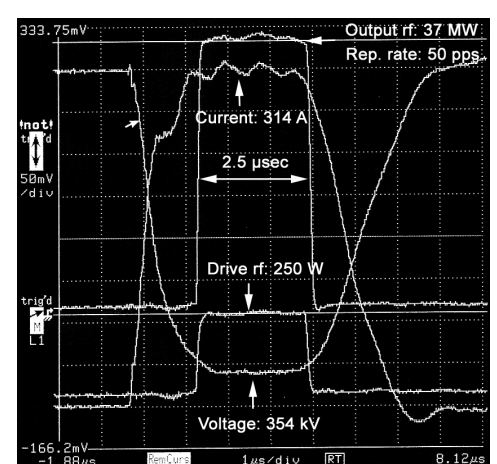

Figure 8: Output rf power, beam voltage and drive rf power waveforms.

\section{DISCUSSIONS}

In the first C-band klystron, the high-power beam has been successfully transported through PPM focusing system, where measured beam-loss was lower than $1 \%$. Therefore, we conclude the designed magnetic circuit and the permanent magnet generated correct PPM field as we expected. It was also demonstrated the HIP process is suitable to fabricate the magnetic circuit in high power klystrons.

A parasitic oscillation was found when the gun-voltage exceeded $320-\mathrm{kV}$. Frequency of the parasitic oscillation is around $5726-\mathrm{MHz}$, which is within the gain-bandwidth. We believe the cause of the oscillation is the back streaming electrons from the beam collector, which supports the positive feedback of rf-signal from the output cavity to the input cavity. This type of parasitic oscillation is sometimes found in conventional solenoid focused klystrons [7]. We are studying the oscillation

mechanism with PIC code, and ray tracing to find out optimum shape of the magnetic field profile around the beam collector. The second Cband PPM klystron is now un-

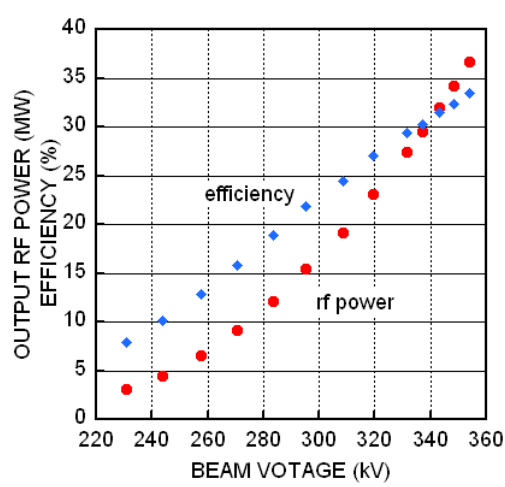

Figure 9: Saturated rf output power characteristics. der design.

\section{REFFERENCE}

[1] Y. Ohkubo et. al., "The C-band 50 MW Klystron Using TravelingWave Output Structure", LINAC98, Aug. 1998, Chicago, IL, USA, KEK Preprint 98-140.

[2] T. Shintake, "C-band Accelerator Development for Linear Collider and Industrial Applications" (invited talk), 23th Linear Accelerator Meeting in Japan, Sep. 1998, Sapporo, JAPAN, KEK preprint 98146.

[3] H. Matsumoto, T. Shintake et. al., "R\&D of The C-band RF-system Development for $\mathrm{e}^{+} \mathrm{e}^{-}$Linear Collider in Japan", 12th Accelerator Science and Technology, Oct. 1999, RIKEN, JAPAN, KEK preprint 99-83.

[4] D. Sprehn, G. Caryotakis, K. Eppley, R.M. Phillips, "PPM Focused X-Band Klystron Development at the Stanford Linear Accelerator Center", (SLAC). SLAC-PUB-7231, Jul 1996. 9pp. 3rd International Workshop on RF Pulsed Power Sources for Linear Colliders (RF 96), Hayama, Japan, 8-12 Apr 1996.

[5] T. Shintake, "Klystron simulation and design the Field Charge Interaction (FCI) cord", Nuclear Instrument and Methods in Physics Research, A 363 (1995) 83-89".

[6] H. Matsumoto et. al., "Applications of the Hot Isostatic Pressing (HIP) for High Gradient Accelerator Structure", PAC'99, May 1991, SF, USA, KEK preprint 91-47.

[7] Z. Fang et al., "Simulation of returning electrons from a klystron collector", Proc. $20^{\text {th }}$ Int. Linac Conference (LINAC200), Monterey, CA, USA, Aug. 2000. 